\section{The better way to prepare nucleic acids}

\author{
- No extraction \\ - No centrifugation
}

- Fast, simple and standardized methods

- High yields of very pure nucleic acids

- Plasmid, M13, PCR ${ }^{1}$ and Oligo Prep Kits available now. More are on the way!

\section{Quick and simple patented method²}

Air from the EasyPrep pump pushes ready made solutions through filters and column wells held in sample plates in the process box. By simply exchanging plates and varying time and pressure, you can complete up to 24

preparations in as little as 30 minutes.

\section{High yieids and purity}

Yields are high. The Plasmid Prep Kit typically recovers $15-20 \mu \mathrm{g}$ double-stranded DNA per $1 \mathrm{ml}$ sample of overnight culture.

Furthermore, products purified with EasyPrep are free from contaminants and are pure enough to use directly in automated sequencing.

\section{EasyPrep - another major advance in nucleic acid preparation.}

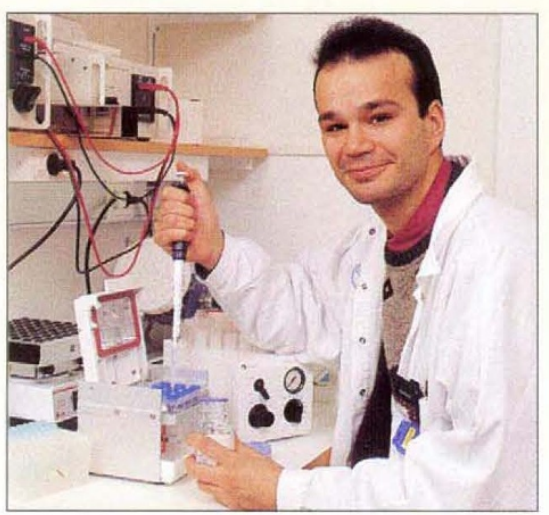

Call your local Sales Office for more details of EasyPrep and EasyPrep Kits.

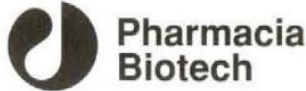

\title{
CNTF in the embryo
}

Sir-We would like to point out that in the article on null mutations in the human CNTF gene by R. Takahashi et al. ${ }^{1}$ there is a small but statistically significant deficit in the number of CNTF null homozygotes, which the authors did not refer to. The expected number of null homozygotes $(\mathrm{M} / \mathrm{M})$ in the total sample $(n=391)$ is 16 compared with an observed frequency of $9\left(\chi^{2}=4.79 ; \mathrm{p}=0.03\right)$, on the assumption of Hardy-Weinberg

Table 1 Comparison of observed versus expected numbers of CNTF genotypes in combined healthy and neurological subjects

$\begin{array}{ccrc}\text { Genotype } & \text { Observed } & \text { Expected } \chi^{2} \\ \text { N/N } & 242 & 249.0 & 0.20 \\ \text { N/M } & 140 & 126.1 & 1.53 \\ \text { M/M } & 9 & 16.0 & 3.06\end{array}$

Calculated gene frequencies for the normal $(N)$ and null $(M)$ alleles, assuming Hardy-Weinberg equilibrium, are 0.798 and 0.202 respectively. The combined $\chi^{2}$ value is $4.79, \mathrm{p}=0.03$.

equilibrium (Table 1). Similar deficits are found within both the normal and neurological control groups. As the numbers are small, this is still a marginal result, but it suggests the possibility that this genotype might be associated with a significantly increased rate of fetal or perinatal mortality. Further studies would be required to confirm the deficit, which although it appears small, could be associated with a very large number of premature deaths. There may be a period during which the developing organism is vulnerable to a deficiency of CNTF, despite the apparent redundancy in growth factors with similar effects.

\section{A. F. Wright}

\section{A. D. Carothers}

MRC Human Genetics Unit,

Western General Hospital,

Crewe Road,

Edinburgh EH4 2XU, UK

IN REPLY - Wright and Carothers point out that the deficit in the CNTF mutant homozygotes might be related to fetal or perinatal death. Considering a large number of premature deaths and the high frequency of mutant homozygotes, their hypothesis seems attractive and plausible. We have already noticed the deficit, although lack of space prevented full discussion.

First, the results of CNTF gene knockout mice do not support the possibility of fetal and perinatal death. Masu and coworkers mated CNTF +/- heterozygotes and determined the genotypes of 98 offspring. They found that the ratio of $\mathrm{CNTF}+/+$, $+/-$ and $-/-$ was about $1: 2: 1$, following Mendelian rules ${ }^{2}$. It has also been demonstrated that CNTF expression in sciatic nerve starts only postnatally ${ }^{3}$. Despite these data, there is still a possibility that CNTF deficiency may be involved in premature death together with other pathological conditions. In this respect, it is noteworthy that a functional CNTF receptor complex (the $\alpha$ component of the CNTF receptor and a leukaemia inhibitory factor (LIF) receptor) is expressed on the surface of embryonic stem (ES) cells and that CNTF, as well as LIF, is able to maintain the pluripotentiality of ES cells ${ }^{4}$. The role of CNTF in embryogenesis remains an intriguing problem.

Second, the population we tested consisted of healthy adult subjects and mainly late-onset neurological disease patients. CNTF deficiency might be a risk factor for an infantile-onset neurological disease or mental retardation, for instance. We are currently continuing analysis of CNTF genotypes in various populations. These studies will clarify the significance of "the deficit" and an unidentified role of CNTF in humans.

\section{Ryosuke Takahashi}

Hidemi Misawa

Department of Neurology,

Tokyo Metropolitan Institute for

Neuroscience,

2-6 Musashidai, Fuchu City,

Tokyo 183, Japan

\section{Takeo Deguchi}

Reseach \& Development Center, BML, Inc.,

1361-1 Matoba, Kawagoe City, Saitama 350, Japan

\footnotetext{
1. Takahashi, R. et al. Nature Genet. 7, 79-84, 215 (1994).

2. Masu, Y. et al. Nature 365, 27-32 (1993).

3. Stöckli, K.A. et al. Nature 342, 920-923 (1989).

4. Conover, J.C. et al. Development 119, 559-565 (1993).
} 